\title{
Dynamics and Energetics of Si Ad-dimers and Ad-dimer Clusters on Ge(100)
}

\author{
W. Wulfhekel, ${ }^{1,2}$ B. J. Hattink, ${ }^{1}$ H. J. W. Zandvliet, ${ }^{1}$ Georg Rosenfeld, ${ }^{3}$ and Bene Poelsema ${ }^{1}$ \\ ${ }^{1}$ Faculty of Applied Physics and Centre of Materials Research, University of Twente, P.O. Box 217, \\ 7500 AE Enschede, The Netherlands \\ ${ }^{2}$ Institut für Grenzflächenforschung und Vakuumphysik, Forschungszentrum Jülich, 52425 Jülich, Germany \\ ${ }^{3}$ Institut für Physikalische und Theoretische Chemie, Universität Bonn, Wegeler Strasse 12, 53115 Bonn, Germany
}

(Received 24 April 1997)

The early stages of silicon heteroepitaxial growth on Ge(100) have been studied with scanning tunneling microscopy. Four different $\mathrm{Si}$ ad-dimer configurations as well as metastable strings of ad-dimers on $\mathrm{Ge}(100)$ have been experimentally identified using dual bias imaging. The metastable strings are aligned along $\langle 130\rangle$ directions and play an important role in binding of $\mathrm{Si}$ atoms and nucleation of Si islands. Various dynamic events, such as the collapse of metastable strings into islands and diffusion events of ad-dimers both along and across substrate dimer rows, have been observed. [S0031-9007(97)04115-X]

PACS numbers: 68.10.Jy, 61.16.Ch, 68.35.Bs, 68.55.-a

In the last decade silicon-germanium heterostructures have received much attention. These heterostructures allow the creation of an entirely new class of materials based on modulated band-gap engineering. It is well established that the growth mode of Ge films on $\mathrm{Si}$ surfaces is of the Stranski-Krastanov type, i.e., that after the formation of a coherent pseudomorphic film with a thickness of a few monolayers 3D cluster formation takes place. Interestingly, the growth of $\mathrm{Ge}$ on $\mathrm{Si}$ is studied in great detailespecially with scanning tunneling microscopy (STM) [1-6], whereas the growth of Si on Ge received much less attention. This is remarkable because both interfaces are of equal importance in SiGe superlattices.

In this Letter we study the initial process of $\mathrm{Si}$ growth on $\mathrm{Ge}(100)$ at room temperature to extend the detailed studies of growth kinetics and nucleation to the second interface present in $\mathrm{Ge} / \mathrm{Si}$ heterostructures. In principle four different isolated and high symmetry adsorption sites for $\mathrm{Si}$ ad-dimers exist on the $(2 \times 1)$ reconstructed Ge(100) surface [see Fig. 1(b) inset]. Using dual bias imaging and STM movies, the different configurations have been identified and from their kinetics and population, conclusions about their energetics and role during nucleation are drawn, showing substantial differences to the growth of $\mathrm{Si}$ on $\mathrm{Si}(100)$. In addition, different diffusion events of ad-dimers have been observed, suggesting a possible kinetic pathway for dimer diffusion across substrate dimer rows.

The experiments were carried out in a UHV chamber (base pressure below $1 \times 10^{-10} \mathrm{mbar}$ ) equipped with a STM and a Si deposition source. The Ge(100) samples were cleaned by cycles of sputtering with $\mathrm{Ar}^{+}$ions and annealing at $1100 \mathrm{~K}$. On clean $\mathrm{Ge}(100)$ the top-layer atoms dimerize, leading to the $(2 \times 1)$ reconstruction. Subsequently, small amounts of $\mathrm{Si}$ were deposited at room temperature. Then, the samples were transferred in situ to the STM for imaging. In order to identify the deposited clusters properly, STM movies at both positive and negative sample bias have been taken. Occasionally we have found rearrangement events in our STM movies which helped us to identify some specific ad-clusters.

In Fig. 1, filled and empty state STM images of the same area of the sample after deposition of $\approx 0.01 \mathrm{ML}$ are shown. The buckled and unbuckled substrate dimer rows of the $(2 \times 1)$-Ge substrate are clearly visible in the filled and empty state image (notice that the observed
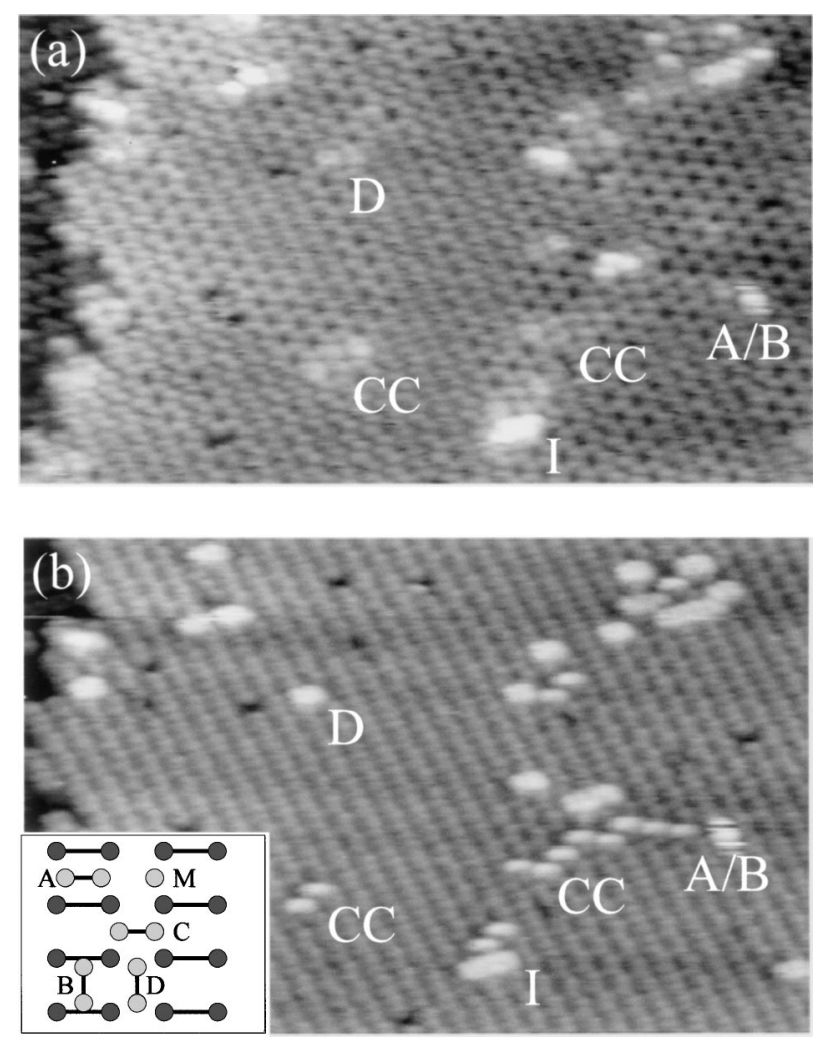

FIG. 1. Filled (a) and empty state (b) images of $\mathrm{Ge}(100)$ after deposition of $\approx 0.01 \mathrm{ML} \mathrm{Si}$ at $300 \mathrm{~K}$. The image sizes are $380 \times 220 \AA$ A. Tunneling parameters: $-1.6 \mathrm{~V}, 1 \mathrm{nA}$ (a); $+1.6 \mathrm{~V}, 1 \mathrm{nA}(\mathrm{b})$. 
buckling alters, when polarity is switched [7]). A variety of different clusters of $\mathrm{Si}$ atoms can be found on the surface. These clusters are absent before deposition. No evidence for single isolated $\mathrm{Si}$ adatoms has been found, suggesting that the deposited $\mathrm{Si}$ adatoms either diffuse easily at room temperature, exhibit a substantial amount of transient mobility, or are moved away by the tip during tunneling. Although at low temperature $(160 \mathrm{~K})$ isolated $\mathrm{Si}$ adatoms and pairs of $\mathrm{Si}$ adatoms coupled via a substrate mediated interaction have been observed on $\mathrm{Si}(100)$ [8], also in the system $\mathrm{Si} / \mathrm{Si}(100)$ the smallest observed isolated features which evolve after deposition at or above room temperature are dimers $[9,10]$.

Similar to molecular-beam epitaxy of $\mathrm{Si}$ on $\mathrm{Si}(100)$ [1] and $\mathrm{Ge}$ on $\mathrm{Si}(100)$ [11], strings of epitaxially oriented dimers, i.e., one dimensional islands (I) of alternating D and $\mathrm{B}$ dimers, are formed that clearly show up in both filled and empty state images. These islands are elongated perpendicular to the substrate dimer rows. Only $19 \%$ of the deposited material is found in these islands at room temperature at this low coverage. When deposition is carried out at elevated temperatures $(400 \mathrm{~K})$ these islands are the predominant species on the surface, similar to the growth of $\mathrm{Si}$ and $\mathrm{Ge}$ on $\mathrm{Si}(100)$ [1,11,12]. Also, smaller bright features A/B, clearly visible in both filled and empty states, are present lying centered on top of a substrate dimer row, which are identified as individual $\mathrm{Si}$ ad-dimers of either type A or B. With $8 \%$ of the total coverage, they play a minor role in condensation of the deposited adatoms. Obviously, these dimers are not static during scanning but seem to be mobile, either thermally or tip induced as can be seen from the strong variation from scanline to scanline. However, the surface is dominated by other features, which only faintly appear in the filled state image, but are clearly visible in the empty state images. These features can be divided into two groups. First, isolated structures of type D are found. In the filled state image, they extend over two substrate dimer rows as a faint disturbance of the dimer rows. In the empty state image, they are centered between two dimer rows. Second, larger features (labeled CC), appearing as strings of faint contrast change in the filled state images, are often seen. In the empty state image, they consist of bright, small features, which are elongated perpendicular to the substrate dimer rows and are centered between the rows of the substrate. Along the dimer rows, they have an extension of roughly one dimer width. They are always separated by one dimer unit perpendicular to the substrate dimer rows and one dimer unit along the dimer rows, i.e., they form strings along the $\langle 130\rangle$ directions.

Figure 2 displays these two types of features in more detail. The isolated feature $\mathrm{D}$ appears round and extends over two dimer rows in the filled state image. The empty state image clearly shows that the structure centered in between the dimer rows is elongated along the rows and hence is identified as a type D dimer. Taking this into
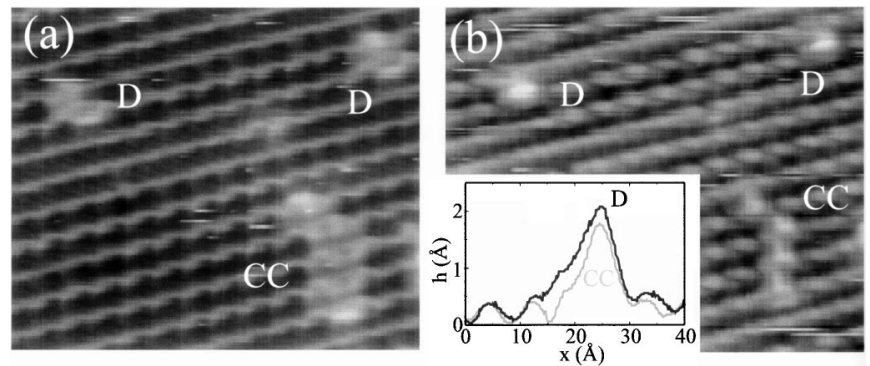

FIG. 2. Filled (a) and empty state (b) images of Si clusters. Size: $150 \times 120 \AA$; Tunneling parameters: $-1.6 \mathrm{~V}, 0.4 \mathrm{nA}$ (b); +1.6 V, $0.4 \mathrm{nA}$ (b). Line scans along the substrate dimer row direction over a $\mathrm{D}$ and $\mathrm{CC}$ cluster centered in between the substrate dimer rows.

account, the filled state image of the D type dimer can easily be explained by a change in the buckling registry of the substrate dimer rows induced by the D dimer, as also visible in Fig. 1(a). The D dimer causes the four adjacent $\mathrm{Ge}$ atoms of the two substrate dimer rows to recede into the surface and the corresponding four other Ge atoms of the four substrate dimers to buckle upwards, resulting in a ringlike feature in the filled state image with a faint additional intensity in the middle caused by the $\mathrm{Si}$ dimer itself. In the case of $\mathrm{Si} / \mathrm{Si}(100)$, only isolated $\mathrm{C}$ and no D dimers have been observed [13], indicating that the D dimers are energetically unfavorable. Obviously, here the system $\mathrm{Si} / \mathrm{Ge}(100)$ differs from $\mathrm{Si} / \mathrm{Si}(100)$. In the case of $\mathrm{Si} / \mathrm{Ge}(100)$, we find $\mathrm{D}$ dimers frequently (39\% of the deposited material). Hence, in contrast to $\mathrm{Si} / \mathrm{Si}(100)$, they play a significant role during nucleation of islands, especially because they have the correct orientation that is needed to form I islands. In addition, after deposition at $350 \mathrm{~K}$, the isolated dimers predominantly present on the surface are $\mathrm{D}$ dimers and annealing of structures deposited at room temperature to $350 \mathrm{~K}$ results in an increase of the population of $\mathrm{D}$ dimers to $\approx 50 \%$ of the deposited material, indicating that they are the dimers of lowest energy.

The stringlike structure $\mathrm{CC}$ cannot be identified that simply. It is clear from the empty state image of Fig. 2 that the strings cannot be explained by clustering of $\mathrm{D}$ dimers, since neither the intensity nor the shape of the individual features of the $\mathrm{CC}$ structure resemble a D dimer. Moreover, the width and height of the individual perturbations along the substrate dimers measured exactly in between two dimer rows are significantly smaller than that of the D type dimer [see Fig. 2(b) inset]. However, the ends of the string in the filled state images look similar to the ringlike features of the $\mathrm{D}$ dimers. It seems to be more likely that the $\mathrm{CC}$ cluster consists of either $\mathrm{C}$ dimers or $\mathrm{Si}$ adatoms that lie in the $\mathrm{M}$ position. To clarify this, we expand our study also to the dynamics of the observed features by taking STM movies at room temperature.

Figure 3 represents an excerpt of such a movie, where during the movie, the polarity of the scan was frequently changed to obtain information in both filled and empty 
states. Because of the frequent switching of polarity, the resolution of the scans is limited, but the surface features can still be clearly identified. First, we focus on a cluster consisting of three small individual parts labeled $\mathrm{CC}_{b}$. During the first half of the movie, i.e., Figs. 3(a)-3(f), the cluster is unchanged. Then, between Figs. 3(f) and $3(\mathrm{~g})$ it collapses and creates two features, a part on the lower side, which remains unchanged during the further scans, and a mobile part positioned on the upper side. In both polarities, the lower part shows up brightly, ruling out a type D dimer. Figures 3(i) and 3(j) clearly show that the part is neither centered on top nor in between the substrate dimer rows, ruling out an A/B dimer. Further it seems that it extends from an on-top position to a position between the substrate dimer rows. Hence, the lower part can be identified as a small island of two epitaxial oriented Si dimers in a row, i.e., a BD structure. According to this, the upper part of the collapsed structure should be a single dimer. In Fig. 3(g) one sees that for one scan it stayed unchanged in the state that it was in the string $\mathrm{CC}_{b}$. Since it is now an isolated feature, the observed shape is likely to be the real shape of the structure and it can be identified as a nonepitaxial dimer lying in between two dimer rows, i.e., a dimer of type C. It certainly can be ruled out to be a type $\mathrm{D}$ dimer, as it clearly distinguishes, e.g., from empty state images of the $\mathrm{D}$ dimer $\mathrm{D}_{\alpha}$ in Figs. 3(a), 3(d), or 3(f). Surprisingly, in the next image [Fig. 3(h)] the $\mathrm{C}$ dimer has diffused to an on-top position (and hence becomes a bright $\mathrm{A} / \mathrm{B}$ dimer) and starts to diffuse along the substrate dimer row. This observation also explains why practically no isolated type $\mathrm{C}$ dimers
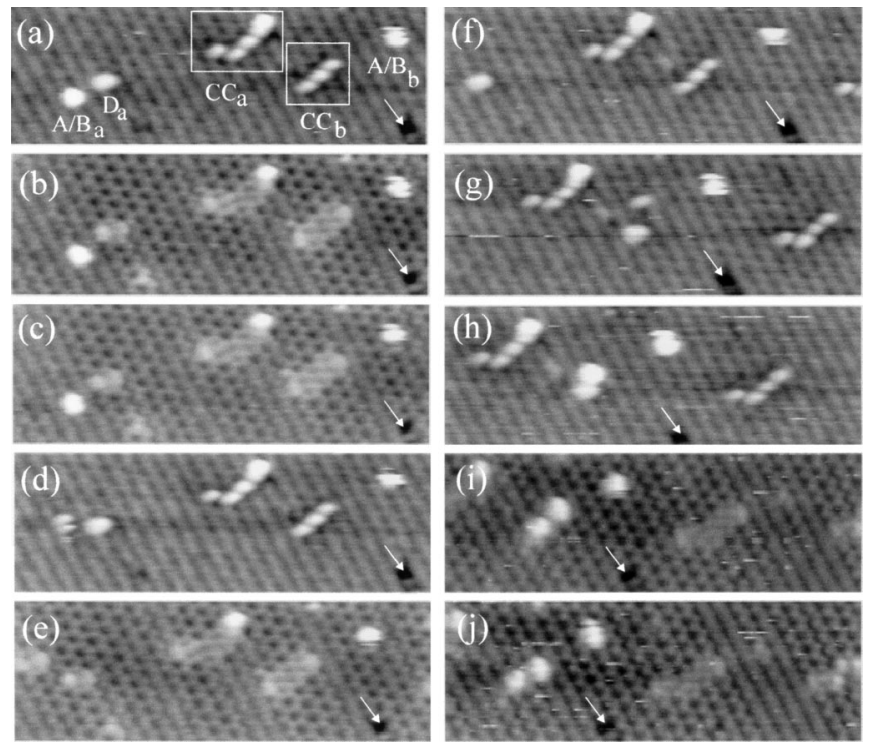

FIG. 3. Successive scans of the same area $(300 \times 100 \AA)$ of filled (b),(c),(e),(i),(j) and empty state (a),(d),(f),(g),(h). Time between images: $150 \mathrm{sec}$. Tunneling parameters: $\pm 1.6 \mathrm{~V}$, $0.4 \mathrm{nA}$. Some drift is illustrated by arrows pointing at an immobile missing dimer defect. are found. They are thermally unstable and transform into A/B dimers and diffuse away or rotate to a more stable D configuration. At room temperature, they can be observed only in clusters, i.e., the CC structure, or very rarely in the vicinity of a missing dimer defect. It can be ruled out that the CC structure is a cluster of single adatoms, since the observed structures after the collapse are incompatible with one dimer and an adatom. The lower part cannot be a dimer and the upper one diffuses only slowly on top of the substrate dimer row in agreement with dimer diffusion and is clearly visible in both states, also characteristic for an A/B dimer in contrast to an adatom [14].

Similar clusters of $\mathrm{C}$ dimers have also recently been seen in the homoepitaxial system of Si [13]. However, the $\mathrm{CC}$ clusters of Si dimers on $\mathrm{Ge}(100)$ are metastable at room temperature and eventually collapse into epitaxially oriented islands as demonstrated in Fig. 3. To clarify how far the observed collapse is thermally activated or tip assisted, we carry out simple annealing experiments. First, 0.02 ML of $\mathrm{Si}$ is deposited at room temperature and STM scans are taken, showing a high density of CC clusters. In a second step, the sample is heated to $350 \mathrm{~K}$ for $5 \mathrm{~min}$. After this short and mild annealing, however, practically no CC structures could be found on the surface anymore. Similarly, after deposition of $0.02 \mathrm{ML}$ of $\mathrm{Si}$ on a sample at $350 \mathrm{~K}, \mathrm{CC}$ clusters are absent on the surface. These observations clearly prove the thermal instability of the CC clusters. In addition, also the kinetic barrier for the collapse can be estimated from these observations. Obviously, $5 \mathrm{~min}$ annealing at $350 \mathrm{~K}$ leads to at least one thermally activated jump per $\mathrm{C}$ dimer. On the other hand, from many STM movies, an upper limit of $\approx 10^{-5} \mathrm{~Hz}$ for the jump frequency at $300 \mathrm{~K}$ can be obtained (less than $\approx 5 \%$ of the CC clusters decay in $2 \mathrm{~h}$ of observation). These upper and lower bounds of the jump frequency allow us to estimate the activation energy $E_{c}$ for the rate limiting diffusion step of the CC cluster collapse as a function of the attempt frequency $\nu_{0}$. Since the two limiting lines for $E_{c}$ defined by the bounding jump frequencies cross when $\nu_{0}$ is varied, it can be concluded that $\nu_{0}$ has to be larger than $\approx 10^{11} \mathrm{~Hz}$ and $E_{c}$ larger than $0.95 \mathrm{eV}$, allowing an error of $10 \mathrm{~K}$ for the annealing temperature. For a typical value of $\nu_{0}$ of $\approx 10^{12.8 \pm 1.3} \mathrm{~Hz}$, as has been observed for Si dimer diffusion on Si [15], $E_{c}$ can be quantified to be $1.1 \pm 0.1 \mathrm{eV}$.

To explain the high concentration of the CC strings (34\% of the deposited material) one has to consider the kinetics of the formation of such structures. Presuming that two adatoms on neighboring rows diffuse along the rows by hopping from $\mathrm{M}$ position to $\mathrm{M}$ position [16] they eventually meet to form a $\mathrm{C}$ dimer. If no more adatoms arrive, the $\mathrm{C}$ dimer will transform into a stable $\mathrm{D}$ dimer or an $\mathrm{A} / \mathrm{B}$ dimer. However, when additional adatoms diffuse along one of the two adjacent substrate dimer rows, it might be trapped in a precursor state one hop away from the $\mathrm{C}$ dimer [13], lowering 
the total energy of the three adatom system and hence stabilizing the system. The addition of a fourth adatom then leads to the formation of a $\mathrm{CC}$ cluster. Because of their large population on the surface, the $\mathrm{CC}$ clusters play an important role in binding the deposited atoms and in nucleation during growth of $\mathrm{Si}$ on $\mathrm{Ge}(100)$. At elevated temperatures, where the $\mathrm{CC}$ clusters become instable on the time scale set by the deposition rate, they may contribute much to the nucleation and formation of $\mathrm{Si}$ islands. In contrast to $\mathrm{Si} / \mathrm{Si}(100)$ [10], no diluted dimer rows have been observed in our case. This suggests that either diluted dimer rows on $\mathrm{Ge}(100)$ are not stable at room temperature or that they are not formed since the state leading to the formation of the CC structure is, due to the different strain field of $\mathrm{Si}$ on $\mathrm{Ge}(100)$ the state of lowest energy.

The movie in Fig. 3 reveals also other information about typical elementary diffusion processes at room temperature. The dimer $\mathrm{A} / B_{b}$ is not static during the scans. It is moving or deforming during the relatively short period of time that it is scanned by the tip, resulting in a ragged appearance. However, from scan to scan, the position of the dimer varies only slightly, although the time between scans is 400 times as long as the time between two scanlines $(0.4 \mathrm{sec} / \mathrm{scanline})$. Therefore, the observed changes in the $\mathrm{A} / \mathrm{B}$ dimer are hardly due to thermal diffusion or displacement by the tip along the dimer row. The behavior can be explained by either a tip induced or a thermal rotation of the dimer between $\mathrm{A}$ and $\mathrm{B}$ orientation as has been observed for Si dimers on $\mathrm{Si}(100)$ on the time scale of seconds to minutes at room temperature $[9,10,15,16]$. It might well be that, following the general trend that the kinetic processes on $\mathrm{Ge}(100)$ have a slightly lower barrier, the observed ragged appearance is due to a thermal activated rotation. In addition to the ragged appearance of the A/B dimer, on longer time scales we observe diffusion of such dimers over large distances along the substrate dimer rows. However, the detailed study of this diffusion process is beyond the scope of this paper.

Besides displacements of $\mathrm{A} / \mathrm{B}$ dimers along the underlying Ge dimer rows also diffusion of dimers across substrate dimer rows has been observed. This diffusion process can be split into two steps. As can be seen in Figs. 3(c)-3(e), the dimer $\mathrm{A} / \mathrm{B}_{a}$ first diffuses along the substrate dimer row and then falls in between the rows to finally become a $\mathrm{D}$ type dimer. In other movies it has been observed that a $\mathrm{D}$ dimer hops to an on-top position (A/B dimer) and then starts to diffuse along the row. Two of these transformation events-A/B to D back to
A/B - may lead to slow diffusion across dimer rows, revealing the kinetic pathway for this kind of diffusion [17]. Hence diffusion of dimers across dimer rows is intimately linked to the transformation between $\mathrm{A} / \mathrm{B}$ and $\mathrm{D}$ dimers. From many STM movies, a lower limit of $0.93 \mathrm{eV}$ for the activation energy of the transformation of an A/B dimer to a $\mathrm{D}$ dimer and hence for diffusion across dimer rows can be given, assuming $\nu_{0}=10^{12.8 \pm 1.3} \mathrm{~Hz}$. An upper limit cannot be given, since we cannot exclude tip effects. The rate for the reverse process, for a D dimer to jump onto a neighboring row, is somewhat lower. This is in accord with annealing experiments to $350 \mathrm{~K}$, after which the concentration of A/B dimers is very low $(<2 \%$ of the deposited material), and the concentration of $\mathrm{D}$ dimers rises. An energy difference between A/B and D dimers, however, cannot be estimated from the annealing experiments, since there may also be some loss of A/B dimers due to diffusion along the substrate dimer rows followed by incorporation at an island, cluster, or step edge. More detailed studies of the energy difference between A/B and D dimers and about their transformation kinetics are needed to quantify dimer diffusion across dimer rows.

G. R. acknowledges support from the Deutsche Forschungsgemeinschaft.

[1] Y.-W. Mo et al., Phys. Rev. Lett. 63, 2393 (1989).

[2] R. J. Hamers, U. K. Köhler, and J. E. Demuth, J. Vac. Sci. Technol. A 8, 195 (1990).

[3] A. J. Hoeven et al., Phys. Rev. Lett. 63, 1830 (1989).

[4] F. Wu, X. Chen, Z. Zhang, and M. G. Lagally, Phys. Rev. Lett. 74, 574 (1995).

[5] M. G. Lagally, Jpn. J. Appl. Phys. 32, 1493 (1993).

[6] Y.-W. Mo et al., Phys. Rev. Lett. 65, 1020 (1990).

[7] J. A. Kubby et al., Phys. Rev. B 36, 6079 (1987).

[8] R. A. Wolkow, Phys. Rev. Lett. 74, 4448 (1995).

[9] Z. Zhang et al., Phys. Rev. Lett. 74, 3644 (1995).

[10] P. J. Bedrossian, Phys. Rev. Lett. 74, 3648 (1995).

[11] Y. W. Mo and M. G. Lagally, Surf. Sci. 248, 313 (1991).

[12] Y.W. Mo et al., Phys. Rev. Lett. 66, 1998 (1991).

[13] J. van Wingerden et al., Phys. Rev. B 55, 4723 (1997).

[14] B. S. Swartzentruber, Phys. Rev. B 55, 1322 (1997).

[15] B. S. Swartzentruber, A.P. Smith, and H. Jónsson, Phys. Rev. Lett. 77, 2518 (1996).

[16] G. Brocks, P. J. Kelly, and R. Car, Surf. Sci. 269, 860 (1992).

[17] After submission of our Letter, a paper by B. Borovsky, M. Krueger, and E. Ganz, Phys. Rev. Lett. 78, 4229 (1997) came to our attention, which deals with dimer diffusion across rows in $\mathrm{Si} / \mathrm{Si}(100)$ via transformation of $\mathrm{A} / \mathrm{B}$ and $\mathrm{C}$ dimers. 